\title{
Heparin Cofactor II in Atherosclerotic Lesions from the Pathobiological Determinants of Atherosclerosis in Youth (PDAY) Study
}

\author{
Jill C. Rau ${ }^{1}$, Carolyn Deans ${ }^{2}$, Maureane R. Hoffman ${ }^{1,3}$, David B. Thomas ${ }^{1}$, Gray T. \\ Malcom ${ }^{4}$, Arthur W. Zieske ${ }^{4}$, Jack P. Strong ${ }^{4}$, Gary G. Koch ${ }^{2}$, and Frank C. Church ${ }^{1,5,6,7}$ \\ ${ }^{1}$ Department of Pathology and Laboratory Medicine, School of Medicine, The University of North \\ Carolina at Chapel Hill, Chapel Hill, NC 27599 \\ ${ }^{2}$ Department of Biostatistics, School of Public Health, The University of North Carolina at Chapel \\ Hill, Chapel Hill, NC 27599 \\ ${ }^{3}$ Pathology and Laboratory Medicine Service, Durham Veterans Affairs, Durham, NC 27710 \\ ${ }^{4}$ Department of Pathology, Louisiana State University Health Sciences Center, New Orleans, LA \\ 70112 \\ ${ }^{5}$ Department of Medicine, School of Medicine, The University of North Carolina at Chapel Hill, \\ Chapel Hill, NC 27599 \\ ${ }^{6}$ Department of Pharmacology, School of Medicine, The University of North Carolina at Chapel Hill, \\ Chapel Hill, NC 27599 \\ ${ }^{7}$ Department of Carolina Cardiovascular Biology Center, School of Medicine, The University of North \\ Carolina at Chapel Hill, Chapel Hill, NC 27599
}

\begin{abstract}
Heparin cofactor II (HCII) is a serine protease inhibitor (serpin) that has been shown to be a predictor of decreased atherosclerosis in the elderly and protective against atherosclerosis in mice. HCII inhibits thrombin in vitro and HCII-thrombin complexes have been detected in human plasma. Moreover, the mechanism of protection against atherosclerosis in mice was determined to be the inhibition of thrombin. Despite this evidence, the presence of HCII in human atherosclerotic tissue has not been reported. In this study, using samples of coronary arteries obtained from the Pathobiological Determinants of Atherosclerosis in Youth (PDAY) study, we explore the local relationship between HCII and (pro)thrombin in atherosclerosis. We found that HCII and (pro) thrombin are co-localized in the lipid-rich necrotic core of atheromas. A significant positive correlation between each protein and the severity of the atherosclerotic lesion was present. These results suggest that HCII is in a position to inhibit thrombin in atherosclerotic lesions where thrombin can exert a proatherogenic inflammatory response. However, these results should be tempered by
\end{abstract}

(C) 2009 Elsevier Inc. All rights reserved.

Address editorial correspondence to: Frank C. Church, Ph.D., Campus Box 7035, 932 Mary Ellen Jones Building, School of Medicine, The University of North Carolina at Chapel Hill, Chapel Hill, NC, 27599-7035 U.S.A., Tel: 01-919-966-3313; Fax: 01-919-966-7639; fchurch@email.unc.edu.

Publisher's Disclaimer: This is a PDF file of an unedited manuscript that has been accepted for publication. As a service to our customers we are providing this early version of the manuscript. The manuscript will undergo copyediting, typesetting, and review of the resulting proof before it is published in its final citable form. Please note that during the production process errors may be discovered which could affect the content, and all legal disclaimers that apply to the journal pertain. 
the additional findings from this, and other studies, that indicate the presence of other plasma proteins (antithrombin, albumin, and $\alpha_{1}$-protease inhibitor) in the same localized region of the atheroma.

\section{Keywords}

Atherosclerosis; immunohistochemistry; heparin cofactor II; thrombin; serpins; PDAY

\section{INTRODUCTION}

Heparin cofactor II (HCII) is a serine protease inhibitor (serpin) that has been strongly implicated in the inhibition of atherosclerosis (Aihara et al., 2007; Aihara et al., 2004; Huang et al., 2007; Tollefsen, 2007; Vicente et al., 2007). Individuals with high levels of HCII have been shown to have less atherosclerosis than their counterparts (Aihara et al., 2004), and HCII deficient mice show increased atherosclerotic plaque formation (Aihara et al., 2007; Vicente et al., 2007). Thrombin exhibits mitogenic and chemotactic activities that contribute to the chronic inflammatory processes of atherosclerosis (Baykal et al., 1995; Harker et al., 1995). Studies that examined restenosis after arterial stent placement showed that elevated blood concentrations of HCII were associated with decreased incidence of restenosis (Schillinger et al., 2004; Takamori et al., 2004); the processes that dominate restenosis, smooth muscle cell proliferation and migration, are also important processes in atherogenesis and can be induced by thrombin. HCII-thrombin complexes have been detected in human plasma (Liu et al., 1995) and, thrombin has been found in its active form in atherosclerotic lesions (Stoop et al., 2000). HCII inhibits thrombin at physiologically relevant rates only in the presence of glycosaminoglycans (GAGs) (Rau et al., 2007). Dermatan sulfate is the predominant antithrombotic GAG in the artery wall (Tovar et al., 2005). It specifically accelerates HCII inhibition of thrombin and has been shown to have decreased activity in atherosclerotic lesions as compared to normal tissue (Shirk et al., 2000). Although HCII is presumed to regulate thrombin in atherosclerosis, the presence of $\mathrm{HCII}$ in atherosclerotic lesions has not been reported.

In this study we utilized atherosclerotic lesions of coronary arteries sampled from the Pathobiological Determinants of Atherosclerosis in Youth (PDAY) research program. PDAY was established in 1985 to quantitatively assess the risk factors for coronary heart disease. Data and arterial samples from over 3,000 individuals from the ages of 15-34 who died of external causes (accidents, homicides, suicides) were collected by fifteen cooperating centers across the United States and managed by the Department of Pathology at Louisiana State University Health Science Center. The data published using PDAY has greatly enhanced our understanding of atherosclerosis and its associated risk factors (see (McGill et al., 2008) for recent review).

We probed the atherosclerotic plaques for HCII, (pro)thrombin and several other proteins with the hypothesis that we would find decreased levels of HCII and increased levels of thrombin in more severe atherosclerotic plaques, reasoning that less HCII would result in decreased thrombin inhibition and therefore more severe atheromas.

\section{MATERIALS AND METHODS}

\section{Histological Samples}

Samples of human left anterior descending (LAD) coronary artery were collected, formalinfixed, paraffin-embedded, serially sectioned and mounted on glass slides by PDAY (Cornhill et al., 1995; Strong et al., 1999; Strong et al., 1997; Wissler, 1994). Twenty-eight cases with varying severity of atherosclerotic lesions were analyzed in this study. 
Sections of formalin-fixed, paraffin-embedded liver were obtained from the McLendon Clinical Laboratory at the University of North Carolina Hospitals, cut into $4 \mu \mathrm{m}$ serial sections and mounted on glass slides. These served as positive controls slides for immunohistological staining as almost all of the proteins that were probed are of hepatic origin. The exception is maspin, a non-plasma serpin of mamillary epithelial origin involved in tumor suppression. Maspin is not synthesized in the liver and thus served as a negative control. A liver section with a maspin-positive tumor served as the maspin-positive control.

\section{Slide Preparation, Mounting and Staining with Hematoxylin and Eosin}

Before staining, paraffin was dissolved and slides were rehydrated using standard techniques. After staining and dehydration all slides were coverslipped using 1 drop of Permount Mounting Media (Fisher Scientific) and air dried overnight. For staining with hematoxylin and eosin, rehydrated slides were immersed first for eight minutes in filtered Mayer's hematoxylin (Dako), rinsed in tap water until no more dye was evident and then soaked in tap water for 10 minutes. Slides were then rinsed in distilled and deionized water before being dipped ten times in $95 \%$ ethanol. Slides were then submersed for 45 seconds in Eosin Y (Sigma) followed by $95 \%$ ethanol for five more minutes.

\section{Antigen Retrieval}

Heat-induced antigen retrieval was employed for probing the AT antigen only. Six slides at a time were placed into plastic Copeland jars with a large hold drilled in the lid filled with Tissue Unmasking Fluid (Invitrogen). These jars were placed into $400 \mathrm{~mL}$ glass beaker in $180 \mathrm{~mL}$ of distilled, deionized water and heated on highest power in a Samsung Classic Collection Microcooking microwave for 2.5 minutes. The water was then exchanged for $180 \mathrm{~mL}$ of fresh water and the beaker containing the Copeland jar with slides was heated again on highest power for 2.5 minutes to bring the internal temperature to $90^{\circ} \mathrm{C}$. The water was exchanged again for $180 \mathrm{~mL}$ of tap water and everything was permitted to cool for 20 minutes.

\section{Immunohistochemical Staining}

Slides were laid flat on a rack and incubated in $460 \mu \mathrm{L}$ per slide of each successive solution. To remove solution, slides were rinsed with Dulbecco's phosphate buffered saline (PBS) (Gibco) containing 1\% Tween 20 (Sigma) $\left(\mathrm{PBStw}_{1 \%}\right)$. Before and between incubation with each solution, slides were washed in $\mathrm{PBStw}_{1 \%}$ for three minutes. First, endogenous peroxidase activity was blocked with either HRP-Block (Dako) for five minutes or with $3 \%$ hydrogen peroxide for ten minutes. Slides were then incubated for one hour in the dark with primary antibody diluted in PBS with either 1\% ovalbumin or 1\% bovine serum albumin. Table 1 describes the antibodies used, their dilutions and incubation times. Next, slides were incubated in the dark with peroxidase conjugated secondary antibody appropriately matched to primary antibody either HRP-conjugated donkey anti-goat $\operatorname{IgG}$ (Serotec) or HRP labeled polymer (Dako). Secondary antibodies were diluted in the same solution as their primary antibodies. Slides were then covered with nine drops per slide of diaminobenzidine solution (DAB) (DAKO) and let sit for eight minutes. DAB staining was enhanced using a solution of 2.5\% cobalt chloride, $2.5 \%$ nickel ammonium sulfate for eight minutes and rinsed in water. Slides were counterstained for five minutes with Mayer's Hematoxylin (Dako or Sigma), rinsed in tap water for five minutes before dehydrating and coverslipping.

\section{Lesion Classification}

One serial section from each case was stained with hematoxylin and eosin. Using this slide set, the severity of each atherosclerotic lesion was classified separately by three trained individuals. Plaque severity was rated from I (least severe) to VI (complicated lesions) according to the American Heart Association classification system (Stary et al., 1995; Stary et al., 1994). 


\section{Staining Intensity}

The intensity of staining for each probed antigen was also independently ranked on a scale of 0 to 3 with 0 indicating no staining, 1 indicating weak staining, 2 indicating intermediate staining and 3 indicating strong staining. Raters were unaware of the specific antigen probed when they were rating staining intensity. Additionally, raters were asked to consider staining intensity only in the tunica intima and tunica media as these are the portions of the vessel susceptible to atherosclerosis. Images were created using the Aperio ScanScope and Aperio ImageScope software version 9.0. Slides were scanned using factory settings for immunohistochemistry.

\section{Statistical Analysis}

Mean scores for lesion severity and staining intensity were computed for each slide. Spearman correlation coefficients, with their corresponding p-values, were computed to evaluate the correlation between the mean scores for each of the probed proteins and severity of the atherosclerotic lesions. The mean score for each protein decreased the variance of scores, thereby increasing the power for detecting a significant correlation when it did exist. The use of the mean scores had support from assessment of intra-rater reliability with intra-class correlations. In order to maintain an overall type 1 error rate of 0.05 throughout five comparisons between lesion severity and staining intensity, there was adjustment for multiple comparisons with the Bonferroni-Holm method; i.e., the smallest p-value was initially compared to $0.05 / 5=0.01$, if significant, then the second-smallest $\mathrm{p}$-value was compared to $0.05 / 4=0.0125$. If significant, the third-smallest $p$-value was compared to $0.05 / 3=0.01667$, and so on for each of the five p-values, stopping when a non-significant result was observed.

\section{RESULTS}

\section{Ratings of Lesion Severity and Antigen Staining of LAD Coronary Artery Sections}

Atherosclerotic lesions were categorized from 1 to 5 . No category 6 lesions were observed. Staining of each of the circulating proteins (HCII, (pro)thrombin, AT, albumin, and $\alpha_{1}$-protease inhibitor) was observed with intensity range of 0 to 3 . Staining intensity for maspin as rated 0 by all reviewers for every section where it was probed despite strong staining on its positive control (tumor in the liver - data not shown). Assessment of inter-rater reliability by inter-class correlation coefficients (ICCs) results in ICCs above 0.5 (Table 2), indicating that there is reliability among raters. This supports the use of the mean scores to assess correlations between plaque severity and staining intensity.

Each of the plasma proteins probed was detected in atheromatous lesions. Maspin was not found in any vessels. Antigen staining was primarily in the lipid rich, necrotic core of the atheromas. Figure 1 shows an example of serial sections of one sample of LAD atherosclerotic coronary artery, immunoprobed for each circulating protein. This atheroma was rated with an average lesion severity of 4.67. Mean staining intensity for the particular atheroma shown in Figure 1 was HCII $=3.00, \mathrm{AT}=1.67,\left(\right.$ pro)thrombin $=3.00, \alpha_{1}$-protease inhibitor $=3.00$, albumin $=3.00$ and goat $\operatorname{IgG}=0$.

It is evident from Figure 1 that there is protein from all of the circulating proteins found in this atheromatous sample. There is some detectable HCII, AT and albumin in the endothelial region, but not the other proteins. It is of value to note the paucity of staining in the less atherosclerotic/ more normal portion of the vessel (seen in the right-most column in Figure 1). No antigen staining was detected in the normal non-atherosclerotic intimal region of this or any of the LAD coronary arteries used in this study. This atheroma represented the pattern of distribution of all of the LAD samples, with staining present predominantly in atherosclerotic core regions and little to no staining perceivable in non-atherosclerotic tunic intima or tunica media. 


\section{Relationship Between Plaque Severity and Antigen Staining}

Spearman correlation coefficients were determined to evaluate the correlation between lesion severity and staining intensity, using the Bonferroni Holm method to adjust for multiple comparisons. This analysis indicates that HCII staining, (pro)thrombin staining, and AT staining were significantly positively correlated with lesion severity (Table 3 ). The relationship between plaque severity and (pro)thrombin was the strongest with its Spearman's coefficient $=0.81$, that for $\mathrm{HCII}=0.63$ and for AT $=0.48$ (Table 3 ). $\alpha_{1}$-Protease inhibitor and albumin did not appear to be correlated with lesion severity (Table 4), although only the most severe slides were available to be rated for these proteins, thus limiting our ability to detect a correlation with lesion severity for these proteins if one does exist.

\section{DISCUSSION}

When beginning the quest to detect the localization of HCII and thrombin in atherosclerotic plaques, we hypothesized that in more severe atherosclerotic plaques there would be low concentrations of detectable $\mathrm{HCII}$ and high concentrations of thrombin as compared with less severe atheroscleromas. Somewhat at odds with this hypothesis, we detected more HCII in the more severe atherosclerotic lesions as evidenced by a statistically significant positive Spearman Correlation (Table 3). However, as predicted (pro)thrombin staining exhibited a positive correlation between intensity and lesion severity. Both HCII and (pro)thrombin were detected in the same lipid-rich regions and necrotic cores of the atheromas. One explanation includes that more thrombin present in severe atheromas drives a more severe atherosclerotic process but also provides more ligand for HCII to bind. To effectively inhibit thrombin, a GAG cofactor is necessary. Dermatan sulfate is the predominant GAG in the arterial wall (Tovar et al., 2005) and is specific for HCII. Furthermore, it has been shown that dermatan sulfate found in atheromas has decreased ability to accelerate HCII inhibition of thrombin (Shirk et al., 1996). Therefore, although there is more HCII in severe plaques than compared to less severe plaques, its ability to inhibit thrombin in this arena may be restricted and thus a more severe plaque is evidenced.

The studies that the inhibition thrombin by $\mathrm{HCII}$ in atherosclerosis, also indicate that $\mathrm{AT}$, the prominent thrombin inhibitor in coagulation, is unable to fulfill this role (Aihara et al., 2007; Vicente et al., 2007). Therefore, to substantiate the functional specificity of HCII in the atheromas, we probed adjacent sections for the presence of AT, expecting to find little there. To our surprise, AT was detected in the atheromas, in the same location as HCII and (pro) thrombin. The AT staining was somewhat less intense across all of the arterial samples despite comparable staining intensity to HCII and (pro)thrombin in the liver positive controls (data not shown). Due to variability in the quality of antibodies and the requirement to use heat-induction for antigen retrieval for AT, it is irresponsible to compare relative protein concentrations for different proteins in these stained vessels. So while the data hints of less AT than HCII in the atheroma, this cannot be firmly established under these conditions. The results do however demonstrate a positive correlation between staining intensity of AT and plaque severity, albeit not as strong as for (pro)thrombin or HCII. This suggested to us that perhaps the HCII and (pro)thrombin co-localization in the lipid-rich core of the atheromas might not be as specific as we had anticipated.

To further investigate the specificity of the co-localization of these thrombin-inhibiting serpins and (pro)thrombin, we probed additional adjacent LAD coronary artery sections for a nonthrombin-inhibiting plasma serpin, $\alpha_{1}$-protease inhibitor $\left(\alpha_{1} \mathrm{PI}\right)$; a non-serpin plasma protein, albumin; and a non-circulating serpin, maspin. Although we can find no evidence establishing a role for $\alpha_{1} \mathrm{PI}$ or albumin in the pathophysiology of atherosclerosis, both of these proteins were found localized in the same region as HCII, (pro)thrombin, and AT (Figure 1). While correlations between staining intensity and plaque severity were not significant with albumin 
and $\alpha_{1} \mathrm{PI}$, this could be due to the smaller number of slides probed. It is perhaps more important that these proteins are found in the atheromas while maspin, which is similarly sized and is from the same family, but is not a circulating protein, was not detected.

In an attempt to determine if the (pro)thrombin in atherosclerotic lesions was thrombin or prothrombin and if it was complexed with either the HCII or AT, we examined homogenized aorta by immunoblot analysis (data not shown). Results from these experiments confirmed the presence of HCII, AT, thrombin and prothrombin in atherosclerotic tissue and also in normal vessel. We did not observe thrombin-serpin complexes in either normal or atherosclerotic homogenized aorta. These results do not eliminate the possibility or even probability that thrombin is inhibited by HCII in the atherosclerotic vessel. It is very likely that serpin-thrombin complexes are cleared more quickly from the vessel microenvironment, or that they are somehow less detectable to the antibodies used here.

The presence in atherosclerotic lesions of all of the circulating proteins probed for in this study, but not the non-circulating serpin, maspin, suggests severe atherosclerotic lesions may act as a mire for plasma proteins and that the co-localization of proteins in this region may be nonspecific. The positive correlation with plaque severity for HCII, (pro)thrombin and AT bolster this theory especially since the endothelium is known to show increased permeability with atherosclerosis (Feletou and Vanhoutte, 2006; Hirano, 2007; Simionescu, 2007). As the barrier function of the endothelium deteriorates, plasma components are more able to filter into the plaque and become trapped in the fatty debris laden region of atheromas. Many other circulating proteins, in addition to those reported here, have been found in the identical region of human atherosclerotic lesions including the apolipoproteins, apoA-I, apoB and apoE (O'Brien et al., 1998; Wyler von Ballmoos et al., 2006), C-reactive protein (Sun et al., 2005), vonWillebrand factor (Sun et al., 2005), and resistin (Burnett et al., 2005). Sound explanations for the existence and retention of these proteins in atherosclerotic plaques have been presented, just as there appears to be good reason for the presence of thrombin and HCII. One could even make a case for the retention of albumin in the plaque as it is known to bind fatty acids. However, we are unable to rationalize the function of $\alpha 1$-protease inhibitor in the atheroma. This is not to say that none of the proteins found in atherosclerotic lesions influence its progression. Contrarily, we find it likely that many of these proteins play a role in atherosclerotic regulation or advancement. For example, fibrin has been found in atherosclerotic plaques (Kaikita et al., 1999). While it is possible that it had been deposited by infiltrating macrophages, it is just as likely that all of the necessary coagulation factors leak into the plaque and are activated by the presence of tissue factor bearing cells in the atheroma. The result would be fibrin in the atherosclerotic plaque that does not resemble a typical fibrin clot because platelets and red blood cells would be absent from the milieu. This hypothesis also explains how thrombin could be detected in its active form in atherosclerotic plaques. Thus the increased permeability of atherosclerotic lesions may actually be a thrombogenic factor in and of itself. This would explain how increased serum HCII protects against atherosclerosis: as serum levels of HCII increase, more HCII crosses into the intimal layer of a vessel with a increased endothelial permeability and is able to neutralize thrombin that has also crossed the endothelium.

Our study also emphasizes a caveat that must be regarded in immunohistochemical examinations of atherosclerosis. While the presence or co-localization of any circulating protein(s) in atherosclerotic lesion may have functionality, it is important to consider that proteins found in the acellular, lipid-rich region of an atheroma, may simply be the result of increased endothelial permeability and may or may not contribute to atherogenesis and to its regulation. 


\section{Conclusions}

In this study, we asked is HCII present in atherosclerotic lesions, does it co-localize with thrombin and is there a relationship between these proteins and the severity of the atherosclerosis? The results of immunohistochemical and immunoblot analyses establish the co-localization of (pro)thrombin with HCII in the core of atherosclerotic plaques and demonstrates a positive correlation between the presence of HCII, (pro)thrombin or antithrombin (AT) and lesion severity. Additionally, these results indicate that atheromas may act as a non-specific watershed for plasma proteins and should serve as a warning against assumptions that co-localization of protein in atheromas implies a pathophysiologic role in atherosclerosis.

\section{Acknowledgments}

Stipend support for J.C. Rau is in part through an NRSA-NIH predoctoral fellowship (1F30AG029053) and previously through the Gertrude B. Elion Mentored Medical Student Award from the Triangle Community Foundation, the UNCCH Integrative Vascular Biology Program NIH grant (NIH, 2T32HL069768), and the Predoctoral Fellowship from the Mid-Atlantic American Heart Association (0715191U). The F.C. Church laboratory is supported in part by the National Institutes of Health (HL-32656). G.T.M., A.W.Z. and J.P.S. were supported in part by the NIH-funded grant "PDAY Cardiovascular Specimen and Data Library" (HL-60808).

\section{REFERENCES CITED}

Aihara K, Azuma H, Akaike M, Ikeda Y, Sata M, Takamori N, Yagi S, Iwase T, Sumitomo Y, Kawano H, Yamada T, Fukuda T, Matsumoto T, Sekine K, Sato T, Nakamichi Y, Yamamoto Y, Yoshimura K, Watanabe T, Nakamura T, Oomizu A, Tsukada M, Hayashi H, Sudo T, Kato S, Matsumoto T. Strain-dependent embryonic lethality and exaggerated vascular remodeling in heparin cofactor IIdeficient mice. J Clin Invest 2007;117:1514-1526. [PubMed: 17549254]

Aihara K, Azuma H, Takamori N, Kanagawa Y, Akaike M, Fujimura M, Yoshida T, Hashizume S, Kato M, Yamaguchi H, Kato S, Ikeda Y, Arase T, Kondo A, Matsumoto T. Heparin cofactor II is a novel protective factor against carotid atherosclerosis in elderly individuals. Circulation 2004;109:27612765. [PubMed: 15148272]

Baykal D, Schmedtje JF, Runge MS. Role of thrombin receptor in restenosis and atherosclerosis. Am. J. Cardiol 1995;75:82B-87-B.

Burnett MS, Lee CW, Kinnaird TD, Stabile E, Durrani S, Dullum MK, Devaney JM, Fishman C, Stamou S, Canos D, Zbinden S, Clavijo LC, Jang GJ, Andrews JA, Zhu J, Epstein SE. The potential role of resistin in atherogenesis. Atherosclerosis 2005;182:241-248. [PubMed: 16159596]

Cornhill JF, Herderick EE, Vince DG. The clinical morphology of human atherosclerotic lesions. Lessons from the PDAY Study. Pathobiological Determinants of Atherosclerosis in Youth. Wien Klin Wochenschr 1995;107:540-543. [PubMed: 7483640]

Feletou M, Vanhoutte PM. Endothelial dysfunction: a multifaceted disorder (The Wiggers Award Lecture). Am J Physiol Heart Circ Physiol 2006;291:H985-H1002. [PubMed: 16632549]

Harker LA, Hanson SR, Runge MS. Thrombin hypothesis of thrombus generation and vascular lesion formation. Am J Cardiol 1995;75:12B-17B.

Hirano K. The roles of proteinase-activated receptors in the vascular physiology and pathophysiology. Arterioscler Thromb Vasc Biol 2007;27:27-36. [PubMed: 17095716]

Huang PH, Leu HB, Chen JW, Wu TC, Lu TM, Yu-An Ding P, Lin SJ. Decreased heparin cofactor II activity is associated with impaired endothelial function determined by brachial ultrasonography and predicts cardiovascular events. Int J Cardiol 2007;114:152-158. [PubMed: 16650906]

Kaikita K, Takeya M, Ogawa H, Suefuji H, Yasue H, Takahashi K. Co-localization of tissue factor and tissue factor pathway inhibitor in coronary atherosclerosis. J Pathol 1999;188:180-188. [PubMed: 10398162]

Liu L, Dewar L, Song Y, Kulczycky M, Blajchman MA, WF J II, Andrew M, Delorme M, Ginsberg J, Preissner KT, Ofosu FA. Inhibition of thrombin by antithrombin III and heparin cofactor II in vivo. Thromb Haemost 1995;73:405-412. [PubMed: 7545318] 
McGill HC Jr, McMahan CA, Gidding SS. Preventing heart disease in the 21st century; implications of the Pathobiological Determinants of Atherosclerosis in Youth (PDAY) study. Circulation 2008;117:1216-1227. [PubMed: 18316498]

O'Brien KD, Olin KL, Alpers CE, Chiu W, Ferguson M, Hudkins K, Wight TN, Chait A. Comparison of apolipoprotein and proteoglycan deposits in human coronary atherosclerotic plaques: colocalization of biglycan with apolipoproteins. Circulation 1998;98:519-527. [PubMed: 9714108]

Rau JC, Beaulieu LM, Huntington JA, Church FC. Serpins in thrombosis, hemostasis and fibrinolysis. J Thromb Haemost 2007;5:102-115. [PubMed: 17635716]

Schillinger M, Exner M, Sabeti S, Mlekusch W, Amighi J, Handler S, Quehenberger P, Kalifeh N, Wagner O, Minar E. High plasma heparin cofactor II activity protects from restenosis after femoropopliteal stenting. Thromb Haemost 2004;92:1108-1113. [PubMed: 15543340]

Shirk RA, Church FC, Wagner WD. Arterial smooth muscle cell heparan sulfate proteoglycans accelerate thrombin inhibition by heparin cofactor II. Arterioscler. Thromb. Vasc. Biol 1996;16:1138-1146. [PubMed: 8792767]

Shirk RA, Parthasarathy N, San Antonio JD, Church FC, Wagner WD. Altered dermatan sulfate structure and reduced heparin cofactor II-stimulating activity of biglycan and decorin from human atherosclerotic plaque. J Biol Chem 2000;275:18085-18092. [PubMed: 10749870]

Simionescu M. Implications of early structural-functional changes in the endothelium for vascular disease. Arterioscler Thromb Vasc Biol 2007;27:266-274. [PubMed: 17138941]

Stary HC, Chandler AB, Dinsmore RE, Fuster V, Glagov S, Insull W, Rosenfeld ME, Schwartz CJ, Wagner WD, Wissler RW. A definition of advanced types of atherosclerotic lesions and a histological classification of atherosclerosis: A report from the Committee on Vascular Lesions of the Council on Arteriosclerosis, American Heart Association. Arterioscler., Thromb., and Vascul. Biol 1995;15:1512-1531.

Stary HC, Chandler AB, Glagov S, Guyton JR, Insull W Jr, Rosenfeld ME, Schaffer SA, Schwartz CJ, Wagner WD, Wissler RW. A definition of initial, fatty streak, and intermediate lesions of atherosclerosis. A report from the Committee on Vascular Lesions of the Council on Arteriosclerosis, American Heart Association. Arterioscler Thromb 1994;14:840-856. [PubMed: 8172861]

Stoop AA, Lupu F, Pannekoek H. Colocalization of thrombin, PAI-1, and vitronectin in the atherosclerotic vessel wall: A potential regulatory mechanism of thrombin activity by PAI-1/ vitronectin complexes. Arterioscler Thromb Vasc Biol 2000;20:1143-1149. [PubMed: 10764685]

Strong JP, Malcom GT, McMahan CA, Tracy RE, Newman WP, Herderick EE, Cornhill JF. Prevalence and extent of atherosclerosis in adolescents and young adults. Implications for prevention from the pathobiological determinants of atherosclerosis in youth study. JAMA 1999;281:727-735. [PubMed: 10052443]

Strong JP, Malcom GT, Oalmann MC, Wissler RW. The PDAY Study: natural history, risk factors, and pathobiology. Pathobiological Determinants of Atherosclerosis in Youth. Ann N Y Acad Sci 1997;811:226-235. [PubMed: 9186600]discussion 235-7

Sun H, Koike T, Ichikawa T, Hatakeyama K, Shiomi M, Zhang B, Kitajima S, Morimoto M, Watanabe T, Asada Y, Chen YE, Fan J. C-reactive protein in atherosclerotic lesions: its origin and pathophysiological significance. Am J Pathol 2005;167:1139-1148. [PubMed: 16192648]

Takamori N, Azuma H, Kato M, Hashizume S, Aihara K, Akaike M, Tamura K, Matsumoto T. High plasma heparin cofactor II activity is associated with reduced incidence of in-stent restenosis after percutaneous coronary intervention. Circulation 2004;109:481-486. [PubMed: 14744972]

Tollefsen DM. Heparin Cofactor II Modulates the Response to Vascular Injury. Arterioscler Thromb Vasc Biol 2007;27:454-460. [PubMed: 17194895]

Tovar AM, de Mattos DA, Stelling MP, Sarcinelli-Luz BS, Nazareth RA, Mourao PA. Dermatan sulfate is the predominant antithrombotic glycosaminoglycan in vessel walls: implications for a possible physiological function of heparin cofactor II. Biochim Biophys Acta 2005;1740:45-53. [PubMed: 15878740]

Vicente CP, He L, Tollefsen DM. Accelerated atherogenesis and neointima formation in heparin cofactor II deficient mice. Blood 2007;110:4261-4267. [PubMed: 17878401]

Wissler RW. New insights into the pathogenesis of atherosclerosis as revealed by PDAY. Pathobiological Determinants of Atherosclerosis in Youth. Atherosclerosis 1994;108:S3-S20. [PubMed: 7802726] 
Wyler von Ballmoos M, Dubler D, Mirlacher M, Cathomas G, Muser J, Biedermann BC. Increased apolipoprotein deposits in early atherosclerotic lesions distinguish symptomatic from asymptomatic patients. Arterioscler Thromb Vasc Biol 2006;26:359-364. [PubMed: 16322531] 

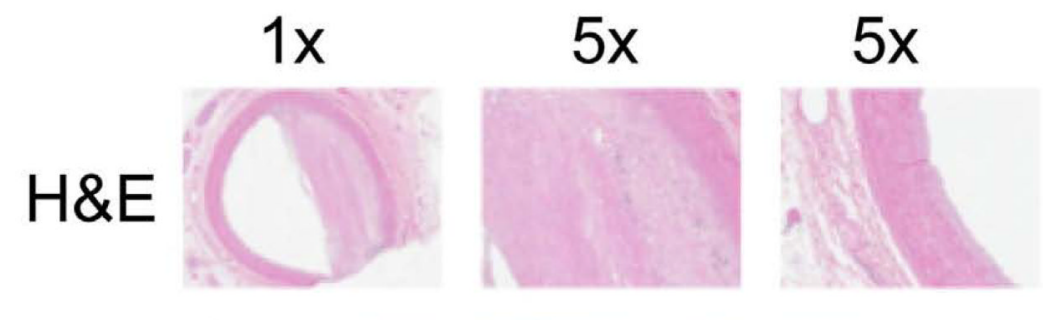

$\mathrm{HCll}$

(Pro)thrombin

AT
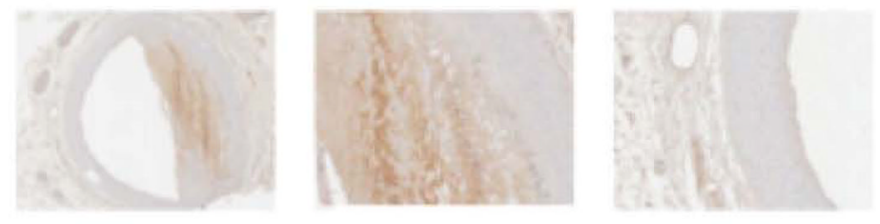

\title{
$\alpha 1-$ Protease Inhibitor
}
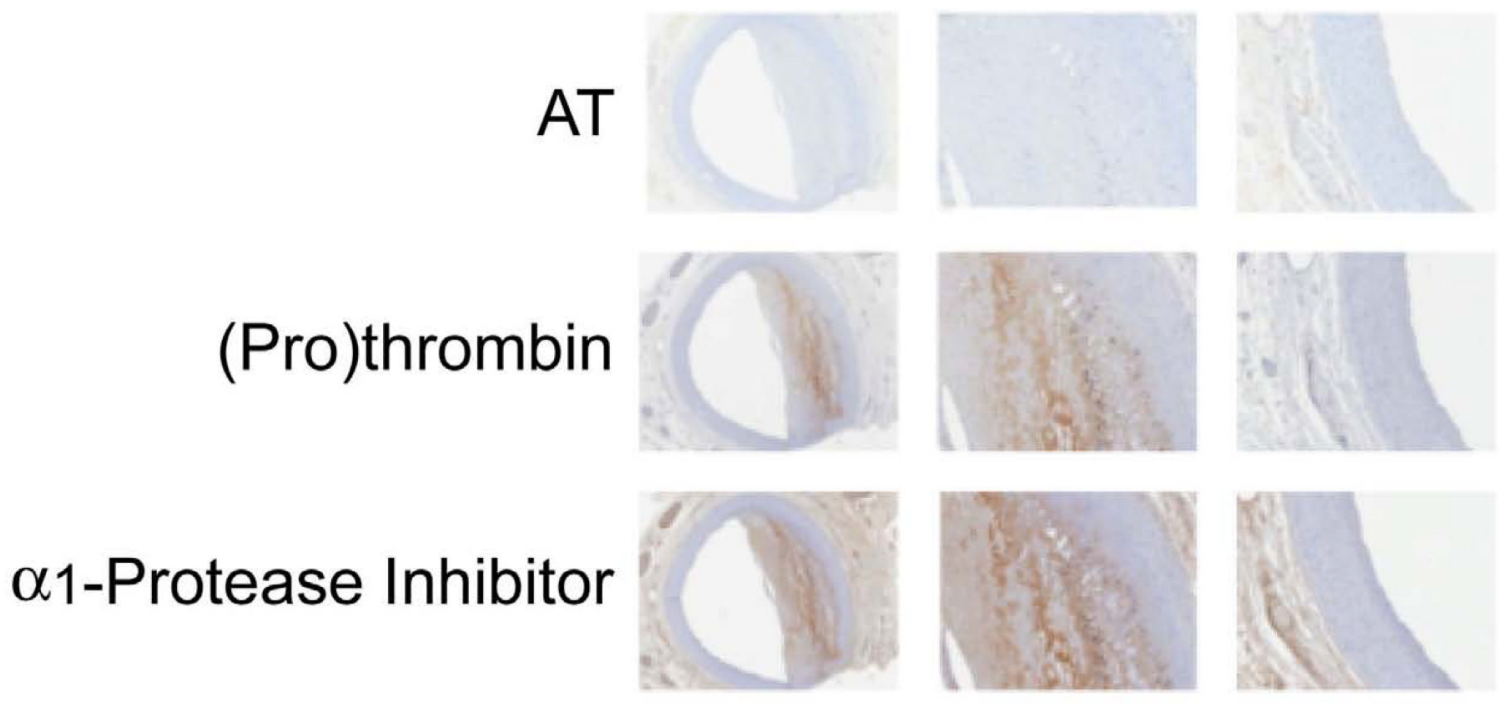

\author{
Albumin
}
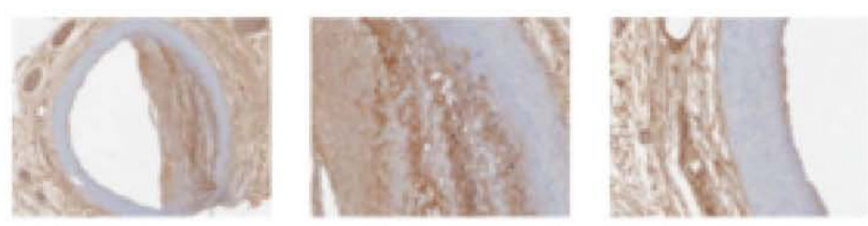

Goat lgG
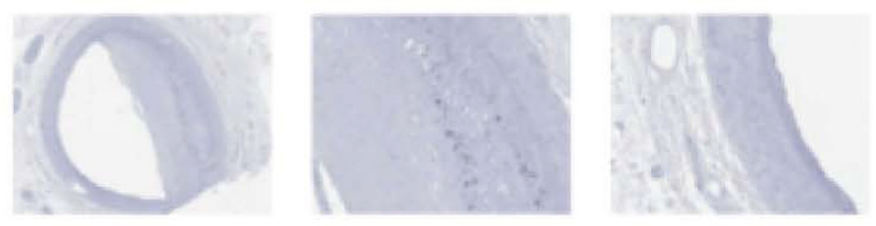

Figure 1. H\&E and immunological staining of an example of a left, anterior descending coronary artery with atherosclerotic lesion for various plasma proteins and goat IgG negative control The left-most column shows a $1 \times$ view of the vessel, the middle column shows a $5 \times$ view of the atheromatous lipid core and the right-most column a $5 \times$ view of the non-diseased region with no visible atherosclerosis. 


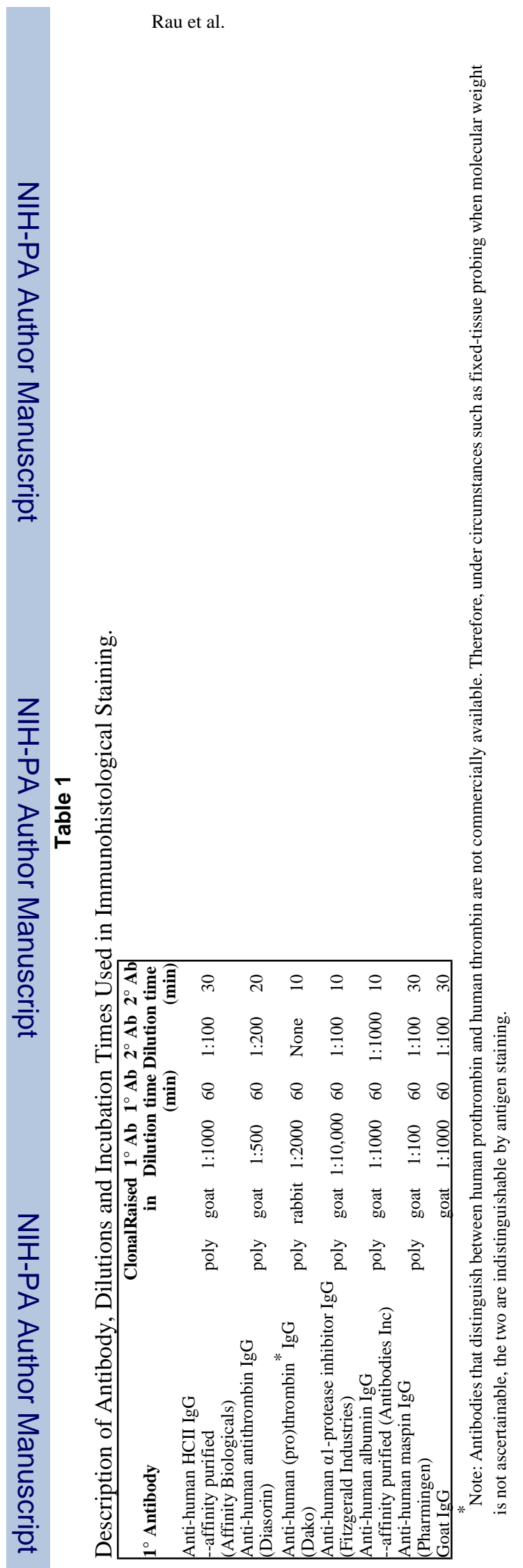

Exp Mol Pathol. Author manuscript; available in PMC 2010 December 1. 
Table 2

Intra-class Correlation Coefficients (ICC) to Address Intra-Rater Reliability

\begin{tabular}{|lr|}
\hline Variable & ICC \\
Severity & 0.824 \\
HCII & 0.705 \\
(Pro)thrombin 0.719 \\
AT & 0.603 \\
a1PI & 0.725 \\
Albumin & 0.845 \\
\hline
\end{tabular}




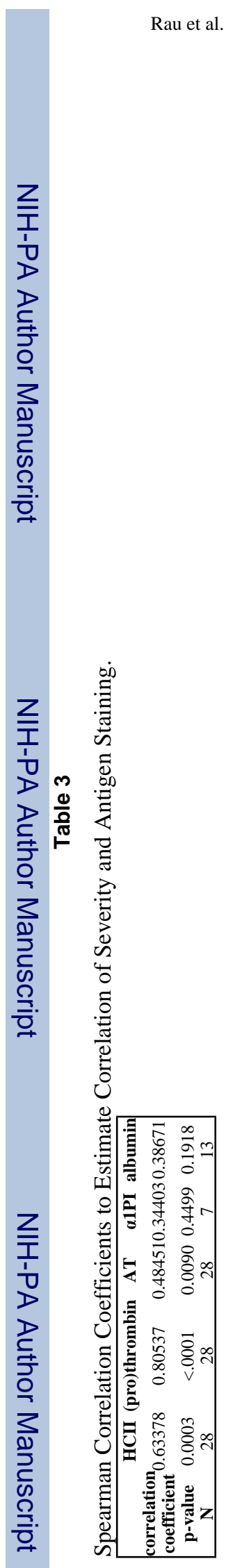

Page 13

Exp Mol Pathol. Author manuscript; available in PMC 2010 December 1. 
Table 4

Breakdown of Tests of Significance Using Bonferroni-Holm Method to Adjust for Multiple Comparisons

\begin{tabular}{|ccccc|}
\hline Step & Variable & Observed p-valueCompare to & Significant? \\
$\mathbf{I}$ & (Pro)thrombin & $<0.0001$ & 0.01 & YES \\
$\mathbf{2}$ & HCII & 0.0003 & 0.0125 & YES \\
$\mathbf{3}$ & AT & 0.0090 & 0.01667 & YES \\
$\mathbf{4}$ & Albumin & 0.1918 & 0.025 & $\mathrm{NO} \rightarrow$ Stop after this step. \\
\hline
\end{tabular}

